\title{
The design of IOV overall structure and the research of IOV key technology in intelligent transportation system
}

\author{
Li Fan ${ }^{*}$, Gangyan $\mathrm{Li}^{1}$, Jian Yang ${ }^{2}$, hongzhi Sun ${ }^{2}$ and Zhanghua Luo ${ }^{2}$ \\ ${ }^{1}$ School of Mechanical and Electronic Engineering, Wuhan University of Technology, Wuhan, \\ 430070, China
}

${ }^{2}$ Air Force Airborne Academy, Guilin, 541003, China

Keywords: Intelligent transportation; Internet of Vehicles; Overall structural design; Key technology.

\begin{abstract}
Internet of Vehicles is the basis of the application of intelligent transportation system .This article has carried on the analysis and discussion about the concept of IOV, applicable architecture, system service type, and need to emphasize solving the key technology of application and development of IOV system. IOV technology involves many disciplines, it needs to be studied further.
\end{abstract}

\section{Introductions}

We solve the traffic problem from two: "road" and "stop”. These two problems can be solved by increasing traffic infrastructu directions re construction, but the cost is high, the cycle is long, the response is slow. Based on the existing foundation conditions, using advanced information technology means to improve traffic conditions is one of the effective measures currently. On the one hand, using the network technology to connect all vehicles can Integrate and share the real-time traffic flow and traffic rate and combine with the traffic condition to guide the vehicles with the purpose of fully excavate the potential of existing roads to improve the efficiency of traffic and road safety, reduce the high cost due to congestion or slow travel of people and the high automobile exhaust emissions; on the other hand, releasing the location of the parking lot and the current spot information to the vehicles terminal by Internet of Vehicles technology can reducing the congestion problems due the drivers' slow cruising and disorderly parking in order to find parking spots.

\section{Concepts and overall architecture of IOV system}

\section{Concepts of IOV system}

In the case of the growth rate of road construction cannot keep up with the vehicles, it is mainly using the vehicle management and reasonable allocation that to solve the congestion problem, namely to establish information system based on car as node-intelligent transportation system based on "Internet of Vehicles". The intelligent transportation is inevitable development direction of the future development; it is a kind of management mode transformation that set the car as the object and the expedition and security as the goal. Vehicles on the road are the main body of traffic; therefore, the basis of intelligent transportation is the "Internet of Vehicles". "Networking" is the synthesis of the existing electronic information technology. Each car is an information node, connect to the intelligent transportation network through wireless communication means, realize the extraction and effective utilization of all attribute information of the vehicle and static and dynamic information on information network platform, and according to the different functional requirements of all the vehicles' running condition provides effective supervision and integrated services, achieves the exchange of information between a car and another car, vehicles and road set, or even the cars and pedestrians and the "dialogue" between vehicles and non-motor vehicles. That will achieve the connectivity of people-car-road and a more intelligent, safer driving experience.

IOV system is a concentrated reflection of the Internet of things technology's application in the 
field of intelligent transportation. IOV system is an essentially part of the Internet of things. Based on the architecture of current Internet of things and the functions that IOV system needed to realize, the architecture of IOV system can be divided into the perception layer, network layer and application layer, which is shown in figure 1.

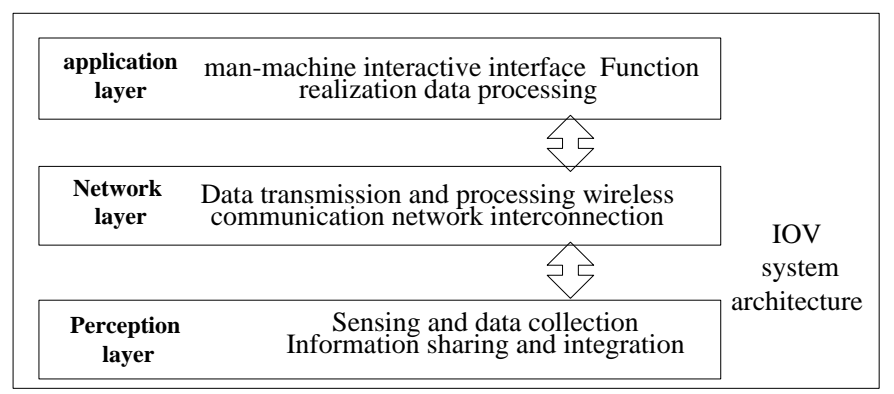

Figure 1.IOV system architecture

Perception layer. Perception layer is IOV's nerve endings, the source of all kinds of information. These sensors can provide vehicles' state information, the related information of the goods they transporting, the state information of the traffic network they are driving in, the path environmental information based on geographic information system, etc. IOV perception layer's main function are the following several aspects: one is using RFID technology for the identification of network nodes, the second one is using a variety of sensors to percept and collect all the data of the people - car road (such as: the intention of driving, the vehicle location, speed, road traffic, weather conditions, etc.), the third one is using the on-board network technology to network all kinds of sensor, realize the data sharing and information fusion.

Network layer. Network layer is the nerve center and brain of the IOV system, its main function is to transmit and process the information obtained from perception layer. Use wireless communication technology to realize the connectivity between nodes and docking with the Internet, complete the analysis and processing of the data and information and transport information over long distance. Network layer is similar to traditional network routers, but have some features that traditional network routers don't have. Such as the bottom protocol used by the IOV system is different from TCP/IP network protocol, so it need network protocol conversion.

Application layer. The application layer mainly dock with the other subsystems, according to the needs of different users provide different applications. The Specific functions include data processing, defining the way of user interaction and realizing the man-machine Interaction. Specific services the IOV system provided are also defined and implemented in this sub-layer. Such as: vehicle running status monitoring, rapid processing of road traffic accident, emergency rescue and dynamic traffic inducing, persuasions and guidance for traffic and parking congestion in the city road, the key vehicle security escort and dangerous goods transportation monitoring, etc.

\section{Network architecture}

IOV's network architecture is mainly composed of vehicle-vehicle communication, vehicle-road communication and vehicle-control center communication. The actual architecture is shown in figure 2. Vehicles use the on-board unit and adjacent vehicles or fixed installations of roadside unit for ad-hoc network communication. On-board unit mainly includes vehicle's running status information collection module, positioning and navigation module, network communication module and the input/output module, etc. Roadside unit is mainly set up near the road, on the one hand, it will send the network traffic manage and control center's information and traffic control command to the nearby vehicle; on the other hand, it will also upload the nearby vehicles' running status information to the traffic control center. On-board unit and intelligent traffic control center also can use the mobile cellular networks for communication, directly receive vehicles' dynamic information and send traffic management instructions. Control center will also summary and processing the acquired vehicle information in its manage area and then selectively provide the data to other services unit, help the service units and other vehicle information service to work. The car drivers and passengers can also use smart phones or other terminals to connect with the vehicle and 
roadside units, acquire the required vehicles and traffic information.

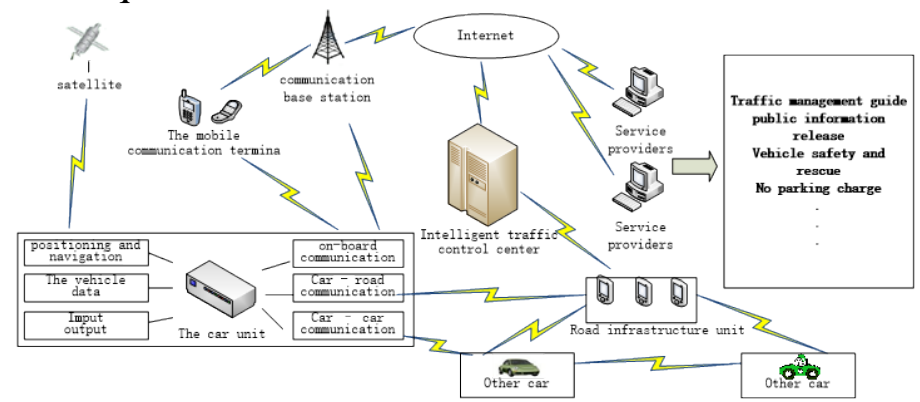

Figure 2.IOV's network architecture

\section{IOV system services types}

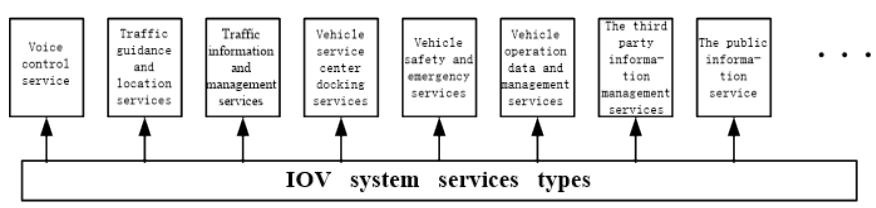

Figure 3.IOV system services

IOV system established in this paper connected the vehicles together to form a network. Based on the network the system can develop more service types and service modes. In this paper, the designed system services are shown in figure 3.

\section{Voice control services}

No matter how good a touch experience is, for the driver, touch the terminal system in the process of driving is unsafe, voice recognition and control technology is particularly important. Therefore. Not only can user connected to the mobile communication network through the networked terminal which is integrated voice module to realize communication services, but also can release all their demand for various kinds of control and service by voice. The service uses the voice recognition module on the terminal for users' instructions' identification, or uploads to the cloud for cloud identification and cloud computing to respond. due to the integrated mobile communication module, in some special situation (such as abnormal startup) the IOV terminal can automatically alarm to owner on the mobile phone which has been set before or send information to the Caution center, realize the anti-rob and anti-theft security services of the vehicle.

\section{Traffic guidance and location services}

IOV system terminal integrated navigation and positioning module which can take advantage of the positioning system and navigation system for vehicle positioning and navigation, with IOV system, it is no longer simply positioning and navigating. Encrypted positioning data can be uploaded to the professional service platform and expand many other services. Especially in the intelligent traffic management area, according to obtained vehicle real-time location information of certain areas can analysis and monitoring traffic flow and data flow. For Area with traffic jams phenomenon, the navigation system may calculate the optimal route to avoid congestion sections in real time according to the latest traffic information service providers provided. The traffic administration can be targeted at vehicles which will be entering the area to send message or dispersion, so as to realize effective management and solve the problem of traffic congestion. At the same time, the unified positioning and statistical analyzed road traffic information can also provide the basis and first-hand data for designing traffic infrastructure planning and road network.

\section{Traffic information and management services}

Intelligent transportation system under the IOV system can realize real time traffic information updating and sharing; provide the data resources for the road traffic participants and managers. In addition to the previously mentioned road traffic management and guidance, access to the parking lot dynamic information can guide the driver whenever they have parking demand; access for 
information of Hotel restaurant service provider can provide booking and guidance services for drivers; such function is very useful Especially for non-local vehicle drivers who are not very familiar with local traffic , avoid the traffic problem that because of the driver is unfamiliar with the road and running slowly on the road for a long period of time looking for bearing.

\section{Vehicle service center docking services}

Many car manufacturers and sellers have already established the corresponding service center, but the service type is based on the Internet or point-to-point voice calls, this way of service need description by speaking or writing so understanding deviation may occur. By using IOV system described in this article, car running dynamic data, such as fault code can be timely transferred to the service provider, thus greatly improve the service quality and service level, it can diagnosis and even start the emergency mode remotely for some fault. In IOV system, vehicle and service center are sat as nodes in a large network structure, this network structure can better guarantee the fastness of related links. In addition, the current service center has certain limitation, such as the vehicles of some brand can only be serviced by their respective company service center, not by each other. But network connectivity is possible in this IOV system. In this mode, vehicles' capacity in the network is elevated, and theoretically the drivers can obtain all services IOV system services provide.

\section{Vehicle safety and emergency services}

Under IOV system, using vehicle sensors acquire vehicle related information and installing related software on the car terminal can detect the vehicle's safety running state online, when malfunction or traffic accidents happen the system can alarm and inform to rescue timely .for example .after a serious traffic accident, even if the drivers and passengers lose consciousness and can't dial telephone, car terminal can automatically dial the emergency telephone or jump send within the network through adjacent vehicles in the same IOV system, and automatically upload the accident vehicle location, vehicle current status and related information. IOV terminal uses wireless communications to realize manual or automatic alarm and emergency rescue call and minimize the loss and casualties caused by road traffic accident due to the slow rescue.

\section{Vehicle operation data and management services}

The attribute information of vehicles along with static and dynamic information is generally stored in automobile electronic control module (ECU), if users want to invoke information (such as vehicle failure) ,they need to read through professional OBD interface by using professional equipment and are unable to prevent the happening of the fault. In default networking system, connecting the IOV terminals to the ECU so it can read related vehicle data at any time. When you need to call, attribute information of the vehicle and static and dynamic information data can be uploaded by IOV to the related service providers, professionals can read information of the state of the vehicle and realize dynamic real-time management to predict faults in time and notify the owner of accordingly operation to avoid or reduce the vehicle fault.

\section{The third party information management services}

Third party information management service involve information, applications and services based on the vehicle provided by a third party. These services are now using RFID card or artificial registration. Under the system of IOV through network connection, vehicles will no longer need to stop for register, taking card, feeing and other related formalities and can directly realize identity authentication, online payment through the IOV. For places with limited permissions, IOV can also complete formalities such as remote access application in advance, which can realize the automatic vehicle access management to save time and improve the level and quality of service.

\section{The public information service}

As we entered the information society, people cannot leave the information services in daily life. IOV connecting with Internet make a lot of Internet applications extended to IOV system. Especially now the $3 \mathrm{~g} / 4 \mathrm{~g}$ mobile network has been completed, which provide the basis of the application for the services demanding greater amount of data.

\section{Key technology of IOV system}

IOV system is network among people, vehicles and road; it is a very large technical system. 
There are many technical and developing problems in application of IOV need to be solved; these problems involve each layer of the IOV architecture.

\section{Radio frequency identification technology (RFID)}

Radio frequency identification (RFID) is a non-contact automatic identification technology, it use radio frequency signal automatically identify targeted objects and access to relevant data without human intervention. At present, RFID technology has been used in many fields, such as entrance guard management, no parking charge system, etc.

RFID applied in IOV system's main advantage is that the technique can identify the high-speed movement of multiple objects; you can easily realize data transmission between the nodes in the IOV. The active RFID technology can provide distance speaking, reading and writing services and can realize active perception. It is suitable for IOV. RFID has advantages of large data storage capacity, small and light, long service life, good waterproof and antimagnetic, safety, etc.

\section{Middleware technology}

Middleware technology is the core technology of the current software research and development. It is very important to pay attention to the development of RFID Middleware in the field of Internet of things. RFID middleware is an intermediate process to implement the data filtering and data format conversion between RFID system hardware devices and application. All kinds of data RFID read-write device read through the process of middleware extraction, decryption, filtration, format conversion and importing car networking applications eventually react on the user interface for the need of IOV users. For different applications of IOV, providers can develop different RFID middleware, such as vehicle routing guide middleware, emergency middleware, traffic information management middleware, etc. Each middleware development needs reference of IOV application service requirements and standards. The middleware technology reduces the difficulty of the application development, improve the development efficiency.

\section{Protocols development}

IOV is suitable for the application category of Internet of things, so it need to research and development the network protocol Suitable for IOV system. Protocol development should also refer to the thought of the OSI network layer, based on vehicle networking architecture and be discussed one layer by another. Research and development of new protocol should bring in the latest research results of the current, combine the actual characteristics of IOV at the same time and pay attention to the efficiency of the agreement. IOV also need access to the Internet, thus would require the study of the problem of IOV protocol conversion makes the data from IOV interflow with the data of the Internet. IOV.

\section{Intellectual technology}

IOV should be a new type of intelligent network. using of smart technology can make the vehicles have some intelligent and be able to actively perceive the change of road environment and real-time traffic conditions and even to predict the drivers' next operation requirements, etc. The main content of intelligent technology research includes the artificial intelligence theory, the intelligent control system, the signal processing and identification and the information fusion, etc.

\section{Safety reliability}

IOV's openness, inclusiveness and anonymity bring some inevitable hidden safe trouble, due to the fast moving of vehicles, changing of driving environmental and frequent access of each node, the car networking application environment is complex and particular, thus puts forward high requirements on its reliability .IOV Should also have the function of defending network attacking, protecting personal privacy and ensuring a fast data transformation.

\section{Conclusions}

Internet of Vehicles is a kind of brand-new concept, it is a new network applications of Internet of things technology in the field of intelligent transportation, the core of a new generation intelligent transportation system and have broad application prospects and commercial value. Hope this article can provide some useful ideas for further study of Internet of Vehicles.

The author's innovative idea in this paper: this paper analyses the concept, Framework and 
application of new Internet of Vehicles, combined with the Internet of things mechanism, applicable system architecture and related key technologies of Internet of Vehicles are discussed in this paper, it may serve as a reference for the further study of Internet of Vehicles.

\section{References}

1.H. Xie, D.C. Dong and D.X. Ou: A New Generation of Intelligent Transportation based on the Internet of Things. Technology \& Economy In Areas Of Communications, (2011) No.13(1)), p.3336,46 .

2.H.X. Zhou: Research on Traffic Information Collection and Processing Methods in Internet of Vehicles (Ph.D., Jilin University, China2013).

3.J.Q.Wang, C.W.Wu and X.J.li: Research on Architecture and Key Technologies of Internet of Vehicles. Control \& Automation, Vol.27 (2011) No.4, p.156-158,130.

4.Z.F.Gu: Vehicle Networking System Architecture and Its Core Technologies (MS., Nanjing University of Posts and Telecommunications, China 2009). 